\title{
Comparison between intermittent mandatory and synchronized intermittent mandatory ventilation with pressure in children
}

\author{
Anne Greenough*
}

\begin{abstract}
Mechanical ventilation can be life saving in critically ill infants and children, but has important complications. As a consequence, new modes of mechanical ventilation have been introduced in an attempt to reduce baro/volutrauma. The modes include those which avoid intubation, such as continuous positive airways pressure (CPAP), minimize excessive volumes, i.e., volume-targeted ventilation
\end{abstract} (VTV) and high-frequency oscillation (HFO), and modes which synchronize the infant's respiratory efforts with ventilator inflations, i.e., patient-triggered ventilation modes. Triggered modes include assist-control ventilation (ACV), when all of the patient's efforts that exceed a critical trigger level are supported by ventilator inflations, and synchronized intermittent mandatory ventilation (SIMV), when a preset number of the infant's breaths trigger ventilator inflations. Pressure support ventilation (PSV) is now also available. During PSV, as with SIMV and ACV, the initiation of the mechanical inflation is determined by the beginning of patient's inspiratory effort but, in addition, termination of inflation is also determined by the patient's inspiratory effort. Exactly when inflation is terminated can be fixed as with the Draeger Babylog ventilator at $15 \%$ of peak inspiratory flow or as with the BIRD-VIP and SLE5000 ventilators it can be manually adjusted (termination sensitivity). Increasing termination sensitivity has been shown to decrease the level of asynchrony, but this was associated with a shorter inflation time, ${ }^{1}$ which could adversely impinge on gas exchange.
There have been physiological, but few randomized, studies assessing the efficacy of PSV compared to other ventilation modes in prematurely born infants. Comparison of SIMV to PSV during four-hour study periods, in 20 infants with a mean gestational age of 29 weeks, demonstrated a reduction in respiratory rate and significant increases in tidal and minute volumes when PSV was used. ${ }^{2}$ It appears that it is important not to use too low a level of PSV. ${ }^{3}$ Comparison was undertaken of two levels of pressure support ( 3 vs. $6 \mathrm{~cm} \mathrm{H}_{2} 0$ ) as an adjunct to SIMV during a $50 \%$ reduction in the SIMV rate, each stage lasting for 30 minutes. ${ }^{3}$ Addition of pressure support (PS) at $6 \mathrm{~cm} \mathrm{H} \mathrm{H}_{2} \mathrm{O}$, but not $3 \mathrm{~cm} \mathrm{H}_{2} \mathrm{O}$, prevented the increased breathing effort seen following a $50 \%$ reduction in SIMV rate. ${ }^{3}$ There have also been comparisons of PSV with volume targeting (VT) to other forms of ventilatory support, those studies ${ }^{4-6}$ have yielded mixed results. In a crossover pilot study, ${ }^{4}$ prematurely born infants in the weaning phase achieved similar oxygenation levels during PSV with VT as with SIMV, but with significantly lower mean airway pressures, suggesting the infants were making a greater contribution to gas exchange during PSV with VT. ${ }^{4}$ Assessment of PSV with VT compared to SIMV as an initial ventilatory mode in prematurely born infants after surfactant treatment for respiratory distress syndrome (RDS) demonstrated that in both groups the peak inspiratory pressure and mean airway pressure (MAP) decreased during the first 24 hours after surfactant administration $(p<0.001)$,

* Division of Asthma, Allergy and Lung Biology, King's College Hospital, London, UK.

Conflicts of interest: Dr. Greenough has received grants and honoraria for lectures from SLE Ltd.

Suggested citation: Greenough A. Comparison between intermittent mandatory and synchronized intermittent mandatory ventilation with pressure in children. J Pediatr (Rio J). 2009;85(1):1-3.

doi:10.2223/JPED.1861 
but the decrease in MAP was faster in the SIMV group ( $p=$ $0.035) .{ }^{5}$ Lung inflammation, as assessed by interleukin levels (IL-1beta, IL-8 and IL-10) before extubation, was higher in infants ventilated with PSV with VT than HFO. ${ }^{6}$

De Moraes et al., ${ }^{7}$ in this issue of the Jornal de Pediatria, report a randomized comparison of intermittent mandatory ventilation (IMV) to SIMV plus PSV in children aged between 28 days and 4 years. Seventy children were randomized; children were excluded if they had undergone tracheostomy or had a chronic respiratory disease. The two groups of children were similar regarding age, indication for mechanical ventilation, pediatric risk of mortality (PRISM) score and a variety of other clinical indices. The researchers found that neither the median time of ventilation, the duration of weaning, nor length of hospital stay differed significantly between the two groups. During weaning the ventilator rate was reduced to 10 bpm. Comparison of SIMV to ACV during weaning of prematurely born infants in three randomized trials ${ }^{8,9}$ demonstrated that ACV was associated with faster weaning: the median duration of weaning was 24 hours vs. 50 hours when the SIMV rate was reduced below 20 inflations per minute. The explanation for those latter findings is that the work of breathing to overcome the resistance of the endotracheal tube was increased when relatively few of the infant's breaths were supported by mechanical breaths. ${ }^{9}$ As the ventilator rate was reduced to $10 \mathrm{bpm}$ in both groups of de Moraes et al.'s study, ${ }^{7}$ this did not bias the results. These results of de Moraes et al.'s study, ${ }^{7}$ however, are in contrast with those of a randomized trial ${ }^{10}$ including 107 extremely low birth weight infants comparing SIMV plus PS to SIMV (although SIMV rather than IMV was the comparator in contrast to de Moraes et al.'s study). In that study ${ }^{10}$ PSV was added at 30 to $50 \%$ of the difference between the positive inflating pressure (PIP) and the positive end-expiratory pressure (PEEP) of the SIMV breaths. A smaller proportion of the infants were still ventilated at 28 days (69 vs. $47 \%, p=0.04$ ) with SIMV and PS than PSV alone; additionally, among infants with a birth weight of 700 to $1,000 \mathrm{~g}$ those supported by SIMV with PSV required fewer days of supplementary oxygen (58 vs. 41 days, $p=0.034$ ). ${ }^{10}$ Those results ${ }^{10}$ suggest that support of every spontaneous breath by mechanical ventilation compared to support of a limited number is advantageous, at least in prematurely born infants. It is possible that in older infants and children, as included in de Moraes et al.'s study, ${ }^{7}$ the work of breathing to overcome the resistance of the endotracheal tube during spontaneous breaths unsupported by mechanical ventilation is less of a problem than in very prematurely born infants, and in children it is less necessary to support every spontaneous breath. In a meta-analysis of the results of randomized trials of ACV or SIMV compared to conventional ventilation (including IMV) in prematurely born infants, use of ACV/SIMV was associated with a significantly shorter duration of ventilation (weighted mean difference 34.8 hours, 95\% confidence interval $62.1-7.4) .{ }^{11}$ From those results, ${ }^{11}$ it might have predicted that a comparison of triggered ventilation (SIMV with PSV) to IMV in children would have demonstrated a shorter duration of ventilation in the children supported by triggered ventilation.

Undertaking randomized studies in the context of the neonatal and pediatric intensive care setting is difficult, but essential if we are to improve the care of these needy patients. Such trials, however, need to be powered to detect clinically important long-term differences.

\section{References}

1. Dimitriou G, Greenough A, Laubscher B, Yamaguchi N . Comparison of airway pressure-triggered and airflow-triggered ventilation in very immature infants. Acta Paediatr. 1998;87: $1256-60$.

2. Migliori C, Cavazza A, Motta M, Chirico G . Effect on respiratory function of pressure support ventilation vs. synchronised intermittent mandatory ventilation in preterm infants. Pediatr Pulmonol. 2003;35:364-7.

3. Osorio W, Claure N, D'Ugard C, Athavale K, Bancalari E. Effects of pressure support during an acute reduction of synchronized intermittent mandatory ventilation in preterm infants. J Perinatol. 2005;25:412-6.

4. Abd El-Moneim ES, Fuerste HO, Krueger M, Elmagd AA, Brandis M, Schulte-Moenting J, et al. Pressure support ventilation combined with volume guarantee vs. synchronized intermittent mandatory ventilation: a pilot crossover trial in premature infants in their weaning phase. Pediatr Crit Care Med. 2005;6:286-92.

5. Nafday SM, Green RS, Lin J, Brion LP, Ochshorn I, Holzman IR. Is there an advantage of using pressure support ventilation with volume guarantee in the initial management of premature infants with respiratory distress syndrome? A pilot study. J Perinatol. 2005;25:193-7.

6. Dani C, Bertini G, Pezzati M, Filippi L, Pratesi S, Caviglioli C , et al. Effects of pressure support ventilation plus volume guarantee vs. high-frequency oscillatory ventilation on lung inflammation in preterm infants. Pediatr Pulmonol. 2006;41: 242-9.

7. de Moraes MA, Bonatto RC, Carpi MF, Ricchetti SM, Padovani CR, Fioretto JR. Comparison between intermittent mandatory ventilation and synchronized intermittent mandatory ventilation with pressure in children . J Pediatr (Rio J). 2009;85:15-20.

8. Chan V, Greenough A. Comparison of weaning by patient triggered ventilation or synchronous intermittent mandatory ventilation in preterm infants. Acta Paediatr. 1994;83:335-7.

9. Dimitriou G, Greenough A, Giffin F, Chan V. Synchronous intermittent mandatory ventilation modes compared with patient triggered ventilation during weaning. Arch Dis Child Fetal Neonatal Ed. 1995;72:F188-90. 
10. Reyes ZC, Claure N, Tauscher MK, D'Ugard C, Vanbuskirk S, Bancalari E. Randomized, controlled trial comparing synchronized intermittent mandatory ventilation and synchronized intermittent mandatory ventilation plus pressure support in preterm infants . Pediatrics. 2006;118:1409-17.

11. Greenough A, Milner AD, Dimitriou G. Synchronized mechanical ventilation for respiratory support in newborn infants. Cochrane Database Syst Rev. 2004;(3):CD000456.
Correspondence:

Professor Anne Greenough

Regional Neonatal Intensive Care Centre

4th Floor Golden Jubilee Wing

King's College Hospital, Denmark Hill

London, SE5 9RS, United Kingdom

Tel.: +44 (20) 3299.3037

Fax: +44 (20) 3299.8284

E-mail: anne.greenough@kcl.ac.uk

\section{Diarrhea mortality: what can the world learn from Brazil?}

\section{Cesar G. Victora*}

Every year, nearly 2 million children all over the world die from diarrhea. In most poor countries, diarrhea is the third most common cause of death of children under 5 years of age, just after neonatal causes and pneumonia. ${ }^{1}$ The annual number of diarrhea deaths worldwide is about the same as that of AIDS deaths at any age - currently estimated at 2.1 million. ${ }^{2}$ Yet, diarrhea attracts much less attention than HIV/AIDS or other currently fashionable diseases such as malaria, that accounts for about 1.3 million deaths a year, at all ages.

In this issue of the Jornal de Pediatria, Melli \& Waldman ${ }^{3}$ use routine data from a Brazilian municipality in the São Paulo metropolitan area to examine recent trends in diarrhea mortality. A strength of their paper is to show how much may be inferred from the judicious use of routine statistical information in an area of southeastern Brazil where vital registration has been complete - or nearly so - for the last few decades.

Their first striking finding is the remarkable drop in diarrhea mortality rates among infants, from 11.9 to 0.2 deaths per 1,000 live births, a 98.6\% reduction between 1980 and 2000. Proportionate mortality data for Brazil as a whole confirm this major decline. Diarrhea was responsible for $17.3 \%$ of all registered infant deaths in 1985-1987, ${ }^{4}$ and by 2003-2005 (the latest year with information) it accounted for $4.2 \%$ of all deaths. ${ }^{5}$ If we take into account that all-cause infant mortality rates for Brazil also dropped from about 60 to just over 20 per 1,000 live births in the same period, the reduction in diarrhea mortality rates per 1,000 live births was roughly $90 \%$. Further support is provided by our studies in
Pelotas, southern Brazil, where the infant mortality rate due to diarrhea fell from 4.2 in 1982 to 0.2 per 1,000 live births in $2004 .{ }^{6}$ Anyone who has worked with child health in Brazil knows that these declines are real. Hospital admissions due to diarrhea have also dropped markedly in the poorest parts of the country, ${ }^{7}$ and it is now difficult, if not impossible, to teach our medical students the signs of acute dehydration in children, which once used to be a common finding in our outpatient and emergency services.

Melli \& Waldman ${ }^{3}$ also report changes in the seasonality of diarrhea, with a marked summer peak associated with bacterial episodes being replaced by a modest peak in the fall, which as the authors argue is likely due to improved sanitation reducing fecal-oral transmission.

However, the most interesting aspect of their analyses of diarrhea deaths is the documentation of reduced geographical disparities, which reflect a decline in socioeconomic inequities. A reduction in the absolute level of a disease is not always associated with a reduction in inequalities - often the opposite seems to occur. ${ }^{8}$ Fortunately, this was not the case in Osasco, state of São Paulo, where the marked decline in mortality led to the virtual disappearance of the geographical disparities. Obviously, if a disease is completely eradicated such as smallpox, polio or measles in Brazil -, social inequalities will disappear as well. This seems to be happening with diarrhea in Osasco.

* Graduate Program in Epidemiology, Universidade Federal de Pelotas (UFPel), Pelotas, RS, Brazil.

No conflicts of interest declared concerning the publication of this editorial.

Suggested citation: Victora CG. Diarrhea mortality: what can the world learn from Brazil? J Pediatr (Rio J). 2009;85(1):3-5.

doi:10.2223/JPED. 1860 